\title{
The microRNA-27a: ZBTB10-specificity protein pathway is involved in follicle stimulating hormone-induced VEGF, Cox2 and survivin expression in ovarian epithelial cancer cells
}

\author{
YUNLI LAI ${ }^{1,2,3^{*}}$, XUERONG ZHANG $^{3,4^{*}}$, ZHENBO ZHANG $^{1}$, YUYAN SHU $^{3,4}$, XIAOLING LUO $^{5}$, \\ YONGBIN YANG ${ }^{1}$, XIAOJIE WANG ${ }^{1}$, GUOPING YANG $^{6}$, LINXIA LI $^{1}$ and YOUJI FENG ${ }^{1}$ \\ ${ }^{1}$ Department of Obstetrics and Gynecology, Shanghai Jiao Tong University Affiliated First People's Hospital, Shanghai; \\ ${ }^{2}$ Genetic and Metabolic Center Laboratory, Guangxi Matemal and Child Health Hospital, Nanning; \\ ${ }^{3}$ Department of Biochemistry and Molecular Biology, Guangxi Medical University, Nanning; ${ }^{4}$ Medical Scientific Research \\ Center of Guangxi Medical University, Nanning; ${ }^{5}$ Department of Medical Research, Guangxi Medical University \\ Affiliated Cancer Hospital, Nanning; ${ }^{6}$ Experimental Research Center, Shanghai Jiao Tong University \\ Affiliated First People's Hospital, Shanghai, P.R. China
}

Received September 24, 2012; Accepted November 23, 2012

DOI: $10.3892 /$ ijo.2012.1743

\begin{abstract}
Previously, we demonstrated that follicle stimulating hormone (FSH) enhanced VEGF expression and facilitated ovarian cancer angiogenesis via the PI3K/AKT signaling pathway. In this study, we further investigated the involvement of microRNA-27a: ZBTB10-specificity protein pathway in the mechanism of FSH-induced VEGF, Cox 2 and survivin expression. Treatment with FSH resulted in significant increase in the expression of VEGF, Cox2, survivin, Sp1 proteins and microRNA-27a in a dose-dependent manner, whereas reverse protein expression pattern was observed in ZBTB10. Downregulation of microRNA-27a using antisense microRNA27a blocked FSH-induced VEGF, Cox 2 and survivin expression. Overexpression of ZBTB10 also attenuated the FSH-induced expression of these molecules. The enhanced expression of VEGF, Cox 2 and survivin was also abolished by knocking down Sp1 using small interfering RNA. Collectively, these results indicated that stimulation of ovarian cancer cell VEGF, Cox 2 and survivin expression by FSH involves the microRNA-27a: ZBTB10-specificity protein pathway.
\end{abstract}

\section{Introduction}

Ovarian cancer is the most lethal gynecological malignancy in women because of occult metastases within the peritoneal

Correspondence to: Dr Linxia Li or Dr Zhenbo Zhang, Department of Obstetrics and Gynecology, Shanghai First People's Hospital, Shanghai Jiao Tong University, 100 Haining Road, 200080 Shanghai, P.R. China

E-mail: zxdzzb@gmail.com

E-mail: zhangzhenbozzb@yahoo.com.cn

*Contributed equally

Key words: microRNA-27a, ovarian epithelial cancer, follicle stimulating hormone, VEGF cavity and the advanced stage at detection when curative therapy is ineffective. Approximately $80-90 \%$ ovarian cancer is origin from ovarian surface epithelium. The etiology of ovarian epithelial cancer (OEC) remains to be clarified, multiple factors involved in OEC development, for example, hormonal, environmental and genetic factors may play a role. Currently, the gonadotropin theory of ovarian cancer proposes that elevated serum gonadotropins, follicle-stimulating hormone (FSH) and luteinizing hormone ( $\mathrm{LH})$, contribute significantly to the development of ovarian cancer. In previous study, Choi et al have reported that FSH enhanced ovarian cancer cells proliferation and invasion by PI3K/AKT signal pathway $(1,2)$. Recent study in our laboratory demonstrated that FSH inhibits ovarian cancer cell apoptosis by upregulating survivin and downregulating PDCD6 and DR5 (3). These studies indicate FSH plays an important role in OEC occurrence, especially in postmenopausal women. In our previous work, it was also reported that activation of the PI3K/AKT pathway mediates FSH-stimulated VEGF expression in ovarian serous cystadenocarcinoma (4). This study also showed that survivin and HIF1 $\alpha$ are involved in FSH-mediated VEGF expression by PI3K/ AKT signal pathway. Survivin is a member of the inhibitor of apoptosis protein (IAP) family, in addition to the anti-apoptosis function, the role of survivin to regulate VEGF expression has been described in various tumor cell types $(5,6)$. These factors contribute to tumor angiogenesis. Inflammation also facilitate tumor angiogenesis, Cox 2 is an important effector molecule of inflammation and was reported to be involve in VEGF expression and tumor angiogenesis. In our recent study, it was found FSH could significantly upregulate Cox 2 expression in a dose-dependent manner (unpublish data). Although these molecules contributing to tumor angiogenesis have positive response to FSH treatment, the detail mechanism and relative signal pathway is not clear.

Mertens-Talcott et al showed evidence that microRNA27a: ZBTB10-specificity protein pathway contributed to breast cancer angiogenesis, decreased microRNA-27a resulted 
in attenuating expression of survivin and VEGF, whereas overexpression of ZBTB10 reduced the protein levels of both molecules (7). MicroRNAs are endogenous 20-25 bp small noncoding RNAs that interact with complementary bingding sites in 3'-untranslated regions of target mRNA to inhibit their expression by blocking translation or enhance mRNA cleavage (8), and play essential roles in a variety of cellular processes, including cell differentiation, proliferation and fat metabolism (9-13). MicroRNA-27a also possesses these oncogenic activities. In addition, microRNA-27a was demonstrated to modulate the cardiac $\beta$-myosin heavy chain gene via thyroid hormone signaling (14). Li et al confirmed that antisense microRNA-27a and overexpression of ZBTB10 blocked estrogen-induced transactivation in breast cancer (15). These studies suggest that microRNA-27a: ZBTB10-specificity protein pathway mediates hormone-induced bio-function. However, the role of this pathway in expression of FSH-induced OEC angiogenesis related molecules has not been addressed. Therefore, in this study, we focused on the roles of microRNA-27a, ZBTB10 and Sp1 in mediating FSH-induced VEGF, Cox 2 and survivin expressions.

\section{Materials and methods}

Chemicals, antibodies, plasmids and reagents. Lipofectamine 2000, DMEM/F12 medium and fetal bovine serum were purchased from Invitrogen. Human follicle stimulating hormone was obtained from Sigma-Aldrich. ZBTB10, SP1, Cox 2, survivin, VEGF, GAPDH and $\beta$-actin antibodies were purchased from Abcam (Cambridge, UK). MicroRNA mirvaRNA extraction kits, the reverse transcription and real-time PCR amplification kits were obtained from Applied Biosciences. As-microRNA-27a (as-miR-27a) was purchased from Applied Biosciences. The ZBTB10 expression plasmid and empty plasmid (pCMV6-XL4) were get from Origene. Sp1 siRNA was obtained from Dharmacon.

Cell lines and cell culture. Human ovarian cancer cell lines, A2780, OVCAR-3, ES-2, HO8910PM, Hey and HO8910, were obtained from the American Type Culture Collection (Manassas, VA) and cultured in 1:1 DMEM/F12. These cell lines were cultured in medium supplemented with $100 \mathrm{U} / \mathrm{ml}$ penicillin, $100 \mu \mathrm{g} / \mathrm{ml}$ streptomycin and $10 \%$ fetal bovine serum. Cultures were maintained at $37^{\circ} \mathrm{C}$ in a humidified incubator containing $95 \%$ room air and $5 \% \mathrm{CO}_{2}$ atmosphere. In addition, the Moody cell line was kindly provided by Dr W. Zheng (Arizona University, Tucson, AZ, USA), it is a normal ovarian epithelial cell line that was transfected with hTERT. The cells were maintained in MCDB109/M199 medium supplemented with $15 \%$ FBS.

Hormone treatment. Ovarian cancer Hey and HO8910 cells were plated in 6-well plates with a cell density of $1 \times 10^{5}$ cells $/ \mathrm{ml}$, respectively. At $60 \%$ confluence, the media were changed to Opti-MEM without serum, starvation for $24 \mathrm{~h}$, the cells were treated with different doses of FSH $(0,25,50$ and $100 \mathrm{mIU} / \mathrm{ml}$ ) for $24 \mathrm{~h}$, the cells were harvested and used for determining the effect of FSH on microRNA-27a expression by real-time PCR. Similarly, both cell lines were treated with different doses of FSH for $48 \mathrm{~h}$, following the cells were collected for western blot analysis to examine the expression patterns of Cox 2, survivin and VEGF protein. To investigate the effects of as-microRNA-27a on FSH-induced VEGF, Cox 2 and survivin, prior to $50 \mathrm{mIU} / \mathrm{ml}$ FSH treatment, Hey and HO8910 cells were treated with as-microRNA-27a, the cells were harvested and the microRNA27a expression was examined by real-time PCR, checked the proteins expression by western blot analysis. In addition, in order to investigate the roles of ZBTB10 and Sp1 on FSH-induced VEGF, Cox2 and survivin expressions, after overexpression of ZBTB10 plasmid or knockdown Sp1, $50 \mathrm{mIU} / \mathrm{ml} \mathrm{FSH}$ was used to treat the cells for another $48 \mathrm{~h}$, the different expression profiles of VEGF, Cox 2 and survivin were analyzed by western blot analysis.

Western blot analysis. The western blot analysis was performed as previously reported. Briefly, after lysis, $60 \mu \mathrm{g}$ proteins were loaded on $10 \%$ SDS-PAGE ges, transferred to polyvinylidene fluoride (PVDF) membranes, and incubated with specific primary antibodies at $4^{\circ} \mathrm{C}$ overnight, followed by 1-h incubation with the appropriate secondary antibody at room temperature, then the bands were visualized with the ECL Plus system (Amersham, GE Healthcare). GAPDH or $\beta$-actin served as a loading control.

Transfection with as-miR-27a and real-time PCR. Transfecion with as-miR-27a was done using siPORT ${ }^{\mathrm{TM}} \mathrm{NeoFX}^{\mathrm{TM}}$ Transfection Agent (Invitrogen) according the manufacturer's instruction. After transfection, the treated cells were harvested and the microRNA was extracted using Taqman microRNA RT kit, followed by amplification using Taqman universal PCR Master mix.

Spl siRNA and ZBTB10 plasmid transfections. siRNA transfections were performed as previously reported, briefly, $1 \times 10^{6}$ cells were seeded in $6-\mathrm{cm}$ dishes and incubated for $24 \mathrm{~h}$, the culture mediums were changed with opti-MEM without serum prior to transfection with $50 \mathrm{nM} \mathrm{Sp1} \mathrm{siRNA.}$ After $12 \mathrm{~h}$, the cells were treated with $50 \mathrm{mIU} / \mathrm{ml} \mathrm{FSH}$ or PBS for another $48 \mathrm{~h}$.

ZBTB10 plasmid transfection was carried out using Lipofectamine 2000 reagent following the manufacturer's protocol. Briefly, after serum starvation for $24 \mathrm{~h}, 4 \mu \mathrm{g}$ plasmid or empty plasmid were mixed with serum-free DMEM/F12 and Lipofectamine 2000 reagent, respectively, following incubated in room temperature for $25 \mathrm{~min}$, then added to the ovarian cancer cells.

Data analysis. Data are presented as the mean \pm standard deviation (SD). The statistical significance of the results was assessed by the Student's t-test or a one way ANOVA using SPSS 11.5 software with $\mathrm{p}<0.05$ being considered significant.

\section{Results}

The expression patterns of microRNA-27a and Spl in normal ovarian epithelial cells and ovarian cancer cells. To examine the possible contribution of microRNA-27a: ZBTB10-specificity protein pathway on ovarian cancer development, we evaluated the expression profiles of microRNA-27a and Sp1 proteins. As illustrated in Fig. 1A, microRNA-27a was overexpressed in 


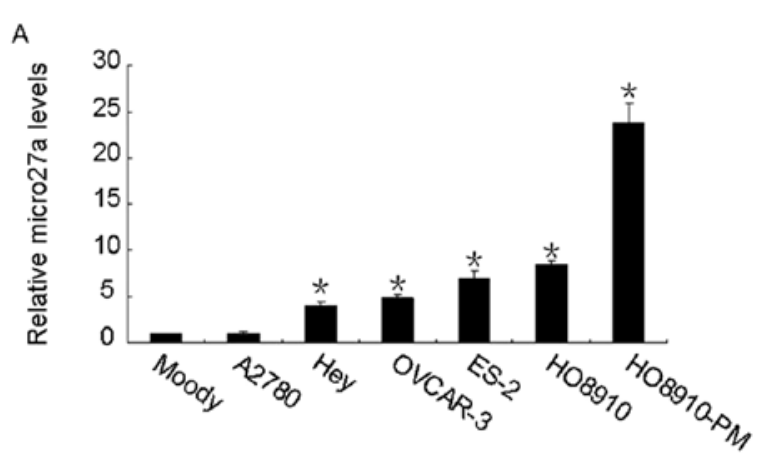

B
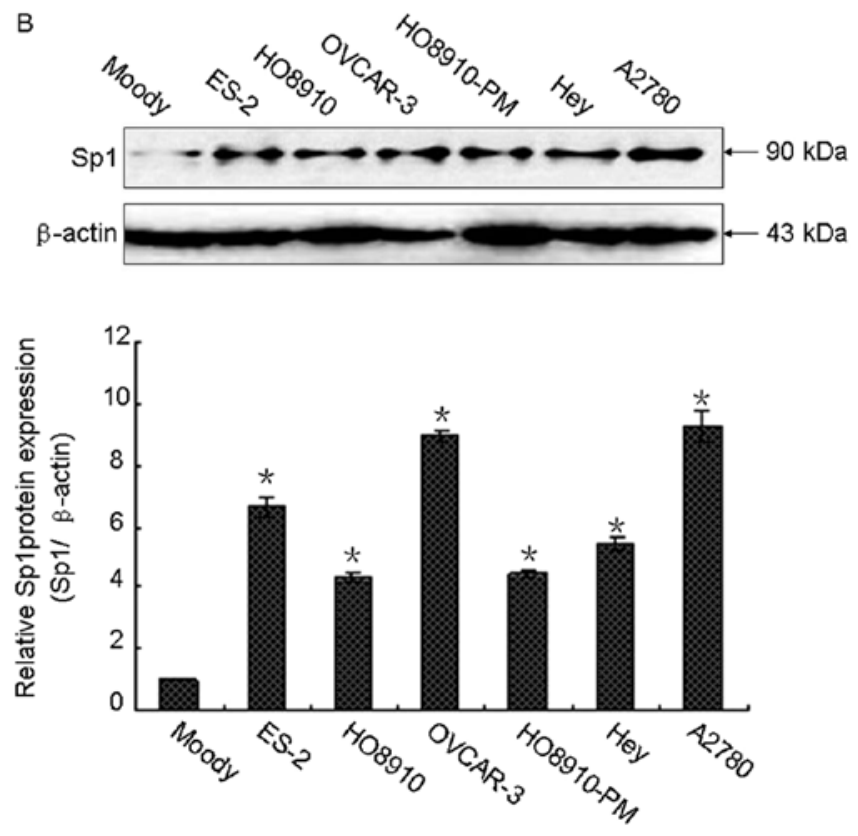

Figure 1. MicroRNA-27a and Sp1 protein are overexpressed in OEC cells (A) MicroRNA-27a expression profile in normal ovarian surface epithelium and ovarian cancer cells was measured using real-time PCR assay. (B) Sp1 protein levels among normal ovarian surface epithelium and ovarian cancer cells were examined using western blot analysis. Data are derived from three experiments and are presented as the mean $\pm \mathrm{SD} ;{ }^{*} \mathrm{p}<0.05$ compared with Moody.

ovarian cancer cells compared with that of Moody cells, which is a normal ovarian epithelial cell line, transfected with hTERT, suggesting that microRNA-27a may play an important role in ovarian cancer development. Similar expression pattern was observed in Sp1, the Sp1 protein levels were higher in ovarian cancer cells than that in Moody cells (Fig. 1B).

FSH activates microRNA-27a: ZBTB10-specificity protein pathway and induces tumor angiogenesis factor expression. In a previous study, we demonstrated that FSH enhanced VEGF expression and promoted angiogenesis (4), however, the detail signal pathway involved in FSH regulation of VEGF and angiogenesis remains to be clarified. Considering that microRNA-27a and Sp1 were overexpressed in ovarian cancer, we further determined whether FSH activated the microRNA27a: ZBTB10-specificity protein pathway. As showed in Fig. 2A, FSH potently enhanced microRNA-27a expression in both Hey and HO8910 cell lines in a dose-dependent manner, the maximal peak of increase of microRNA-27a was observed when the cells were treated with $50 \mathrm{mIU} / \mathrm{ml} \mathrm{FSH}$ for $24 \mathrm{~h}$. Moreover, western blot analysis demonstrated that FSH treatment resulted in significantly increased expression of survivin, Cox2, VEGF and Sp1 (Fig. 2B and C), on the contrary, it showed a reverse expression pattern in ZBTB10 protein, decreasing ZBTB10 protein was observed accompanying the increasing dose of FSH (Fig. 2C).

Inhibition of microRNA-27a blocks FSH-activating microRNA-27a: ZBTB10-specificity protein pathway and abolishes FSH-induced tumor angiogenesis factor expression. To investigate the role of microRNA-27a on FSH-induced tumor angiogenesis factor expression, inhibition of microRNA-27a was performed using as-miR-27a. As showed in Fig. 3A, transfection of as-miR-27a obviously decreased micro-27a expression in both Hey and HO8910 cell lines. As illustrated in Fig. 3B, transfection of as-miR-27a induced approximately a 2-fold (Hey) or 1.5-fold (HO8910) increase of ZBTB10, the effect was abolished by $50 \mathrm{mIU} / \mathrm{ml} \mathrm{FSH}$ treatment for $48 \mathrm{~h}$. However, transfection of as-miR-27a resulted in a 10-16-fold decrease of Sp1, 7-9-fold decrease of Cox2, 2-3-fold decrease of survivin, 2-3-fold decrease of VEGF. Moreover, the FSH-induced Sp1, Cox2, survivin and VEGF expression were blocked by transfection of as-miR-27a (Fig. 3B and C).

Overexpression of ZBTB10 blocks microRNA-27a: ZBTB10-specificity protein pathway and FSH induces tumor angiogenesis factor expression. To analyse the role of ZBTB10 on FSH-induced tumor angiogenesis factor expression, overexpression of ZBTB10 was performed. As showed in Fig. 4A, transfection with ZBTB10 significantly enhanced ZBTB10 protein expression, whereas FSH treatment attenuated this effect. As expected, overexpression of ZBTB10 potently inhibited Sp1, survivin, Cox 2 and VEGF expression in both Hey and HO8910 cell lines, moreover, the increased expression induced by FSH also was attenuated (Fig. 4A and B).

Knockdown of Spl abolishes microRNA-27a: ZBTB10specificity protein pathway and FSH induces tumor angiogenesis factor expression. To test whether Sp1 is involved in FSH-induced tumor angiogenesis factor expression, knockdown of Sp1 was performed by siRNA. Transient transfection of Sp1 siRNA obviously blocked Sp1 protein expression in both Hey and HO8910 cell lines (Fig. 5A). In addition, it resulted in decreasing of Cox2, survivin and VEGF levels. Moreover, FSH-induced Cox2, survivin and VEGF expressions were abolished by knocking down siSp1 (Fig. 5B).

\section{Discussion}

Based on the gonadotrophin theory, the hormone environment is an important factor for ovarian cancer occurrence, especially in postmenopausal women due to increased FSH and LH levels resulting from loss of feedback of estrogen. Currently, aberrant FSH level is considered as a high risk factor for OEC development. Except for enhancing OEC cell proliferation, migration and invasion, and blocking of apoptosis, increasing evidence indicates that FSH induces angiogenesis which is a pivotal step in ovarian cancer development, growth, and invasion beyond the regional border $(4,16,17)$. VEGF is a glycoprotein 
A

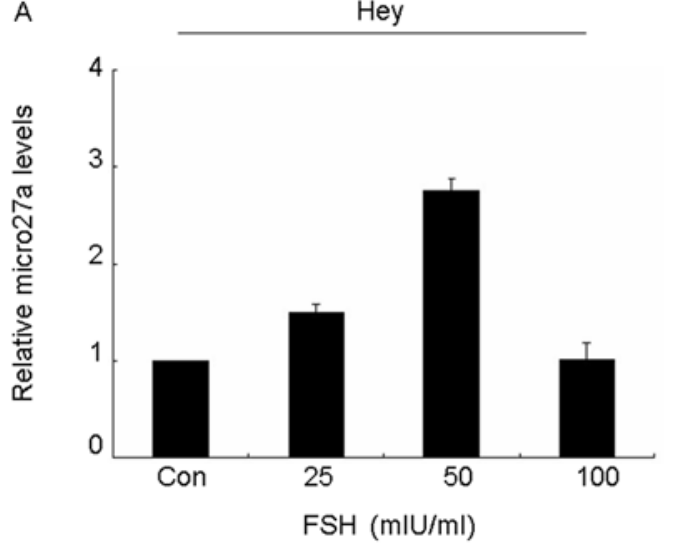

B
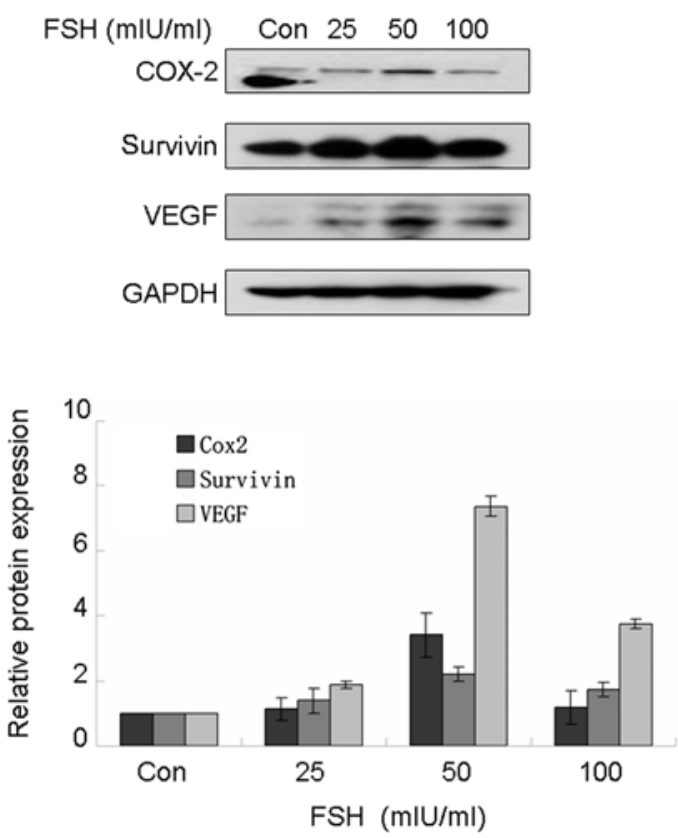

C
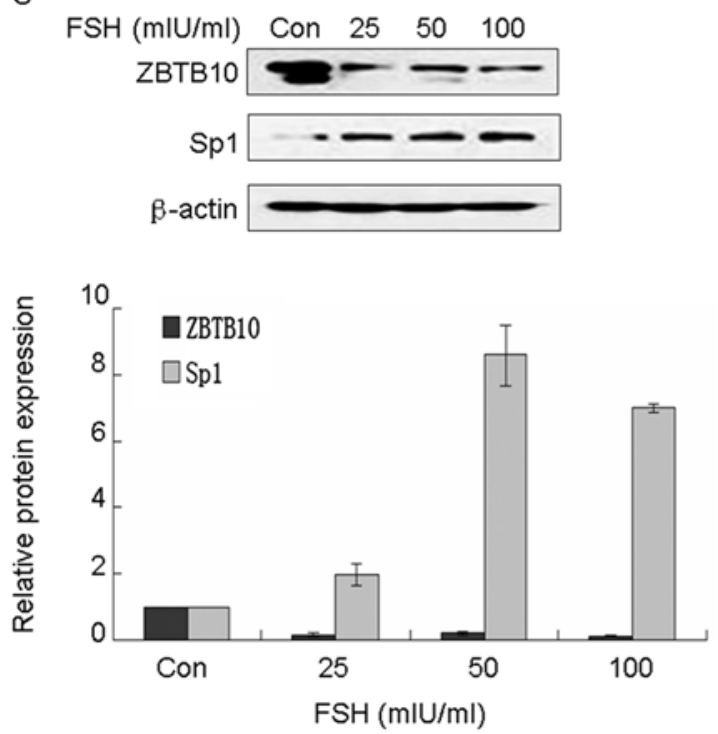
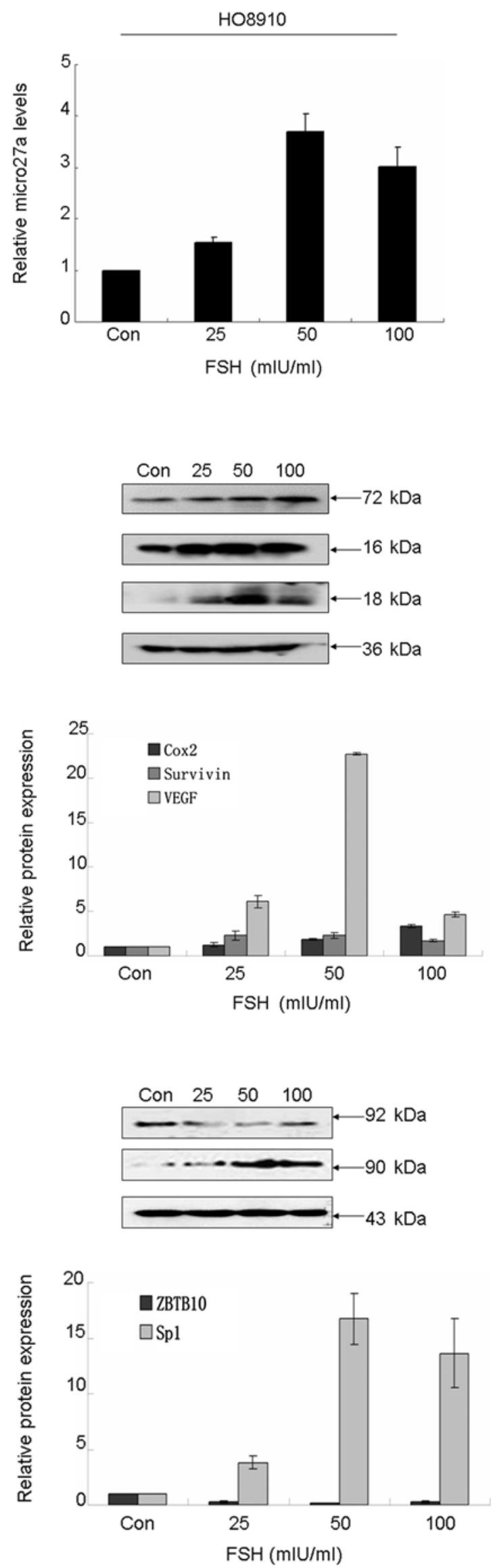

Figure 2. FSH activates micoRNA-27a: ZBTB10-specificity protein pathway and upregulates VEGF, Cox2 and survivin. (A) FSH induced microRNA-27a expression in a dose-dependent manner, and was determined by real-time PCR in both Hey and HO8910 cells. (B) The protein levels of VEGF, Cox 2 and survivin were measured by western blot analysis after treatment with various doses of FSH. (C) The expression patterns of ZBTB10 and Sp1 were detected by western blot analysis after treatment with various doses of FSH. 
A

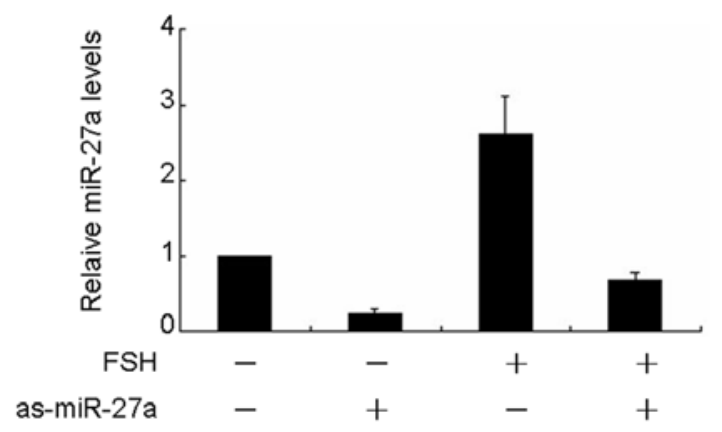

B
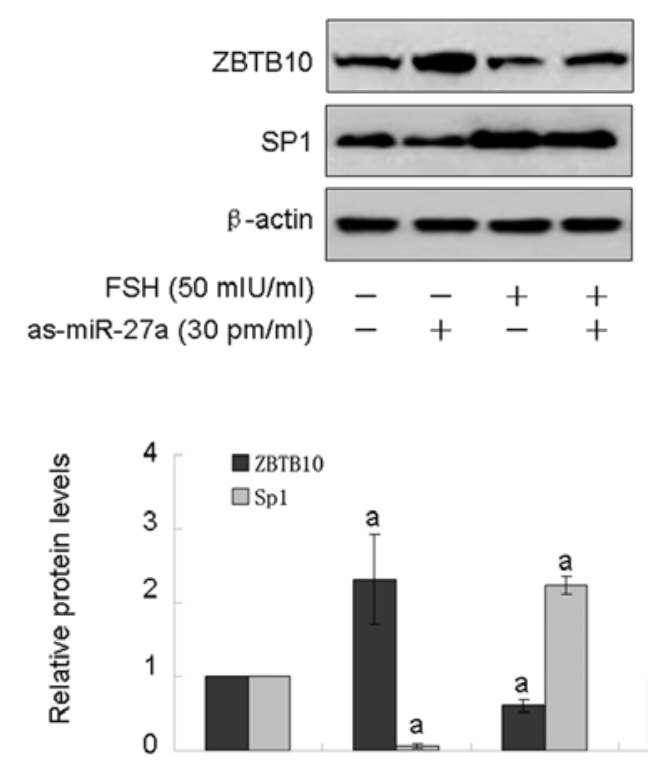

$\mathrm{FSH}(50 \mathrm{mlU} / \mathrm{ml})$

as-miR-27a $(30 \mathrm{pm} / \mathrm{ml})$

C
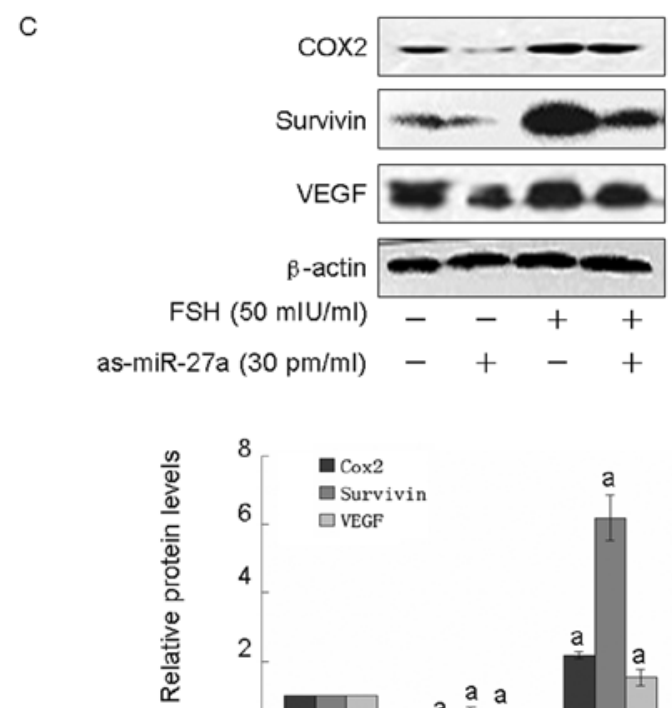

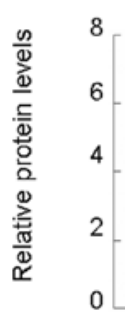

FSH $(50 \mathrm{mlU} / \mathrm{ml})$

as-miR-27a $(30 \mathrm{pm} / \mathrm{ml})$
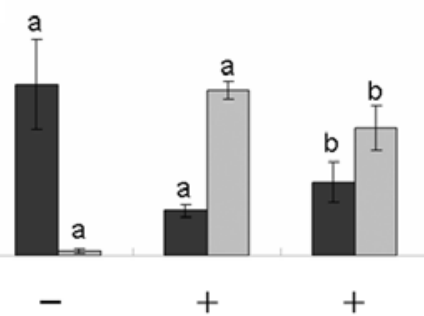
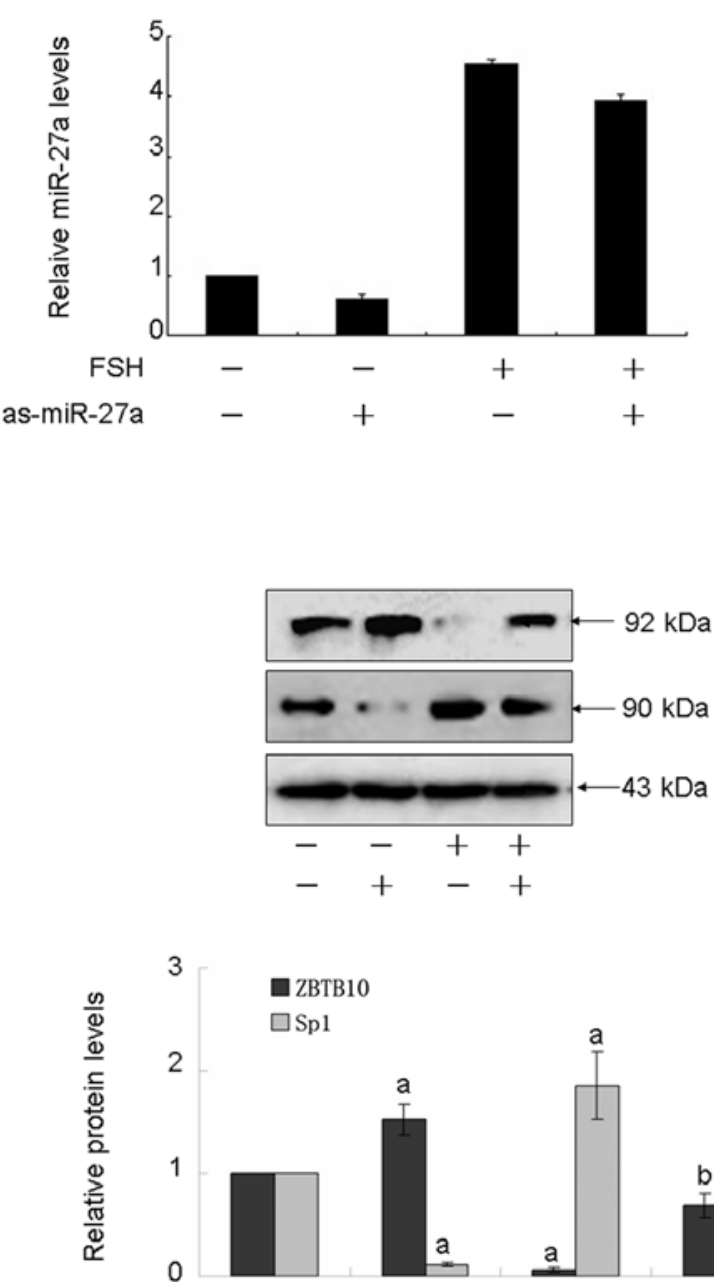

$\mathrm{FSH}(50 \mathrm{mlU} / \mathrm{ml}) \quad-\quad+\quad-\quad+$ as-miR-27a $(30 \mathrm{pm} / \mathrm{ml}) \quad-\quad \ldots \quad+\quad+$

Figure 3. Involvement of microRNA-27a (miR-27a) in FSH-induced VEGF, Cox2 and survivin expression via ZBTB10 and Sp1. (A) Inhibition of transfection of as-miR-27a- induced microRNA-27a expression was measured by real-time PCR assay. The effects of the decrease of microRNA-27a plus 50 mIU/ml FSH treatent on (B) ZBTB10 and Sp1, (C) VEGF, Cox 2 and survivin were determined by western blot analysis. Each experiment was repeated three times. ${ }^{\mathrm{a}} \mathrm{p}<0.05$ compared with control group (no as-miR-27a and no FSH treatment); ${ }^{\mathrm{b}} \mathrm{p}<0.05$ compared with FSH-treated group. The left column presents the results of Hey cell line, the right presents the results of HO8910 cells. 
A
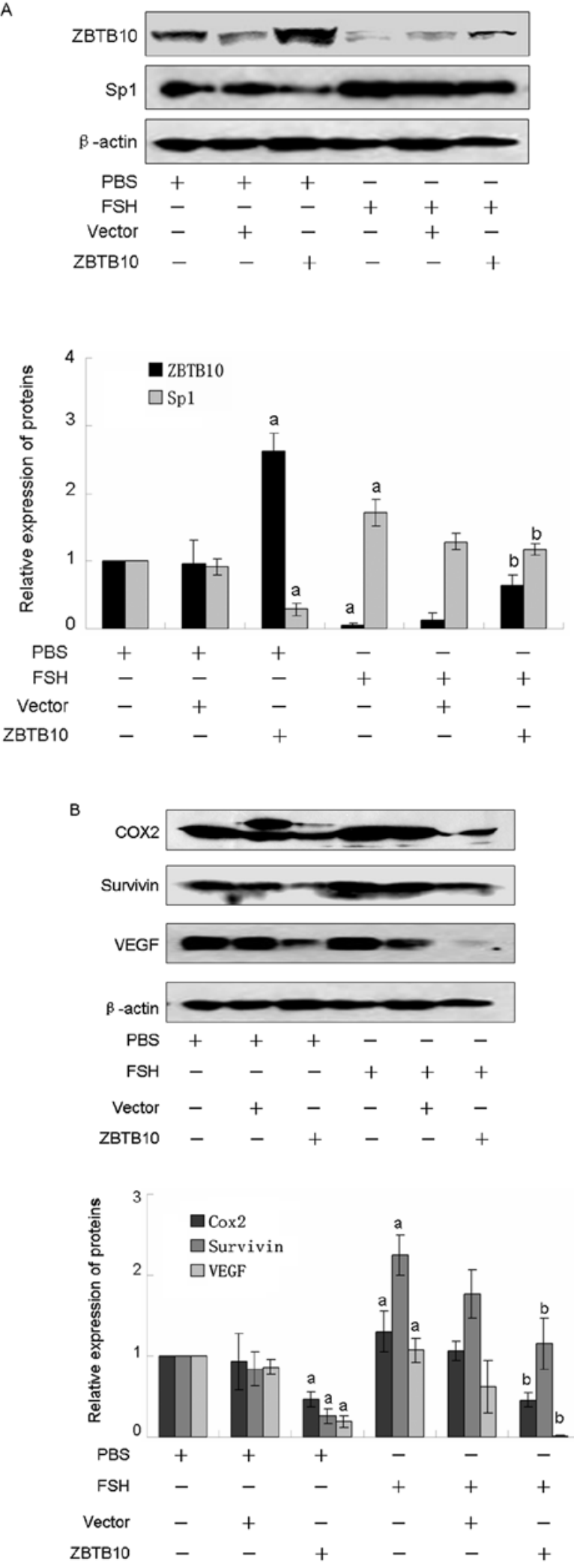
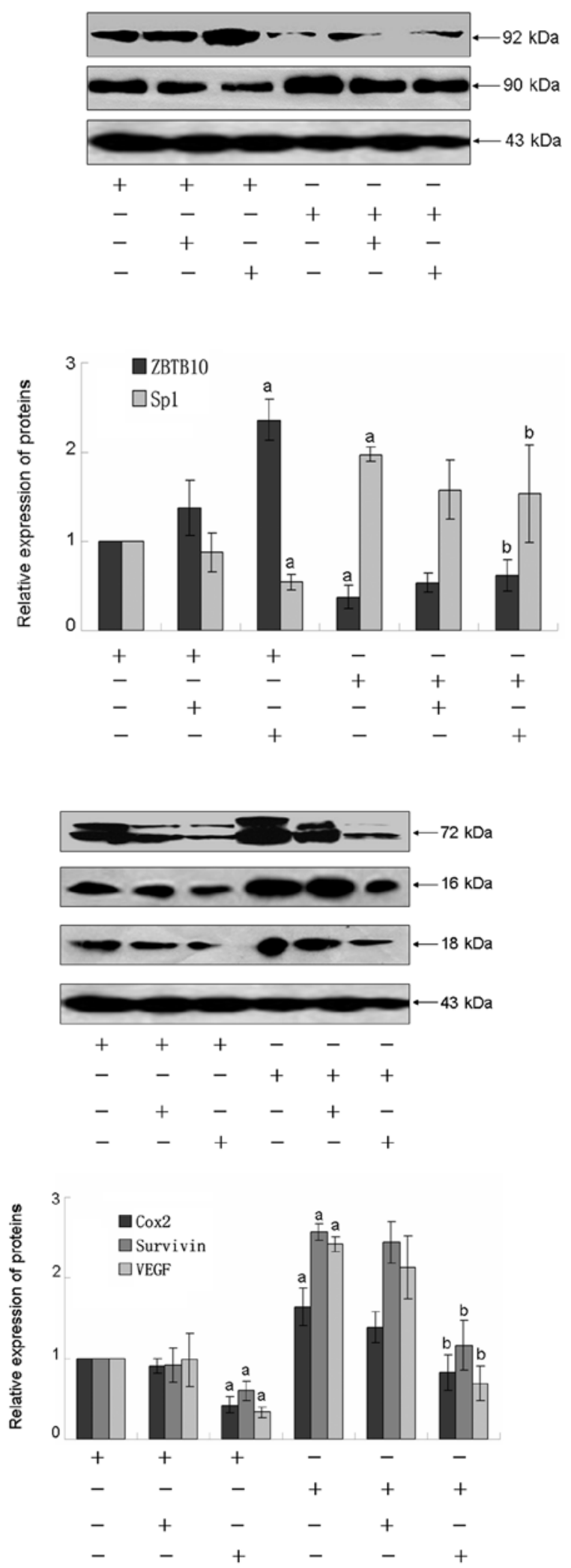

Figure 4. Overexpression of ZBTB10 blocks FSH-induced VEGF, Cox2 and survivin expression. Hey (left column) and HO8910 (right column) cells were transfected with ZBTB10 expression plasmid and empty vector using Lipofectamine 2000, respectively, after transfection for $12 \mathrm{~h}$, the cells were treated with PBS or $50 \mathrm{mIU} / \mathrm{ml} \mathrm{FSH} \mathrm{for} \mathrm{another} 48 \mathrm{~h}$, western blot analysis was performed to measure (A) ZBTB10, Sp1 and (B) VEGF, Cox 2 and survivin expression. ${ }^{\mathrm{a}} \mathrm{p}<0.05$ compared with control group (no ZBTB10 expression plasmid transfection and no FSH treatment); ${ }^{\mathrm{b}} \mathrm{p}<0.05$ compared with ZBTB10-overexpression cells.

which is associated with tumor angiogenesis, higher levels of VEGF are consistent with ovarian cancer poor prognosis and tumor progression (18). In our previous study, it was found that FSH enhanced VEGF expression mediated by survivin 
A
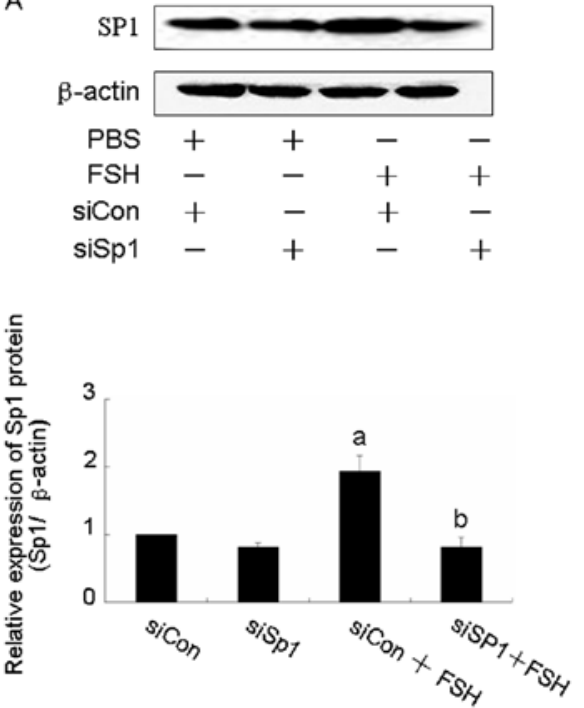

B
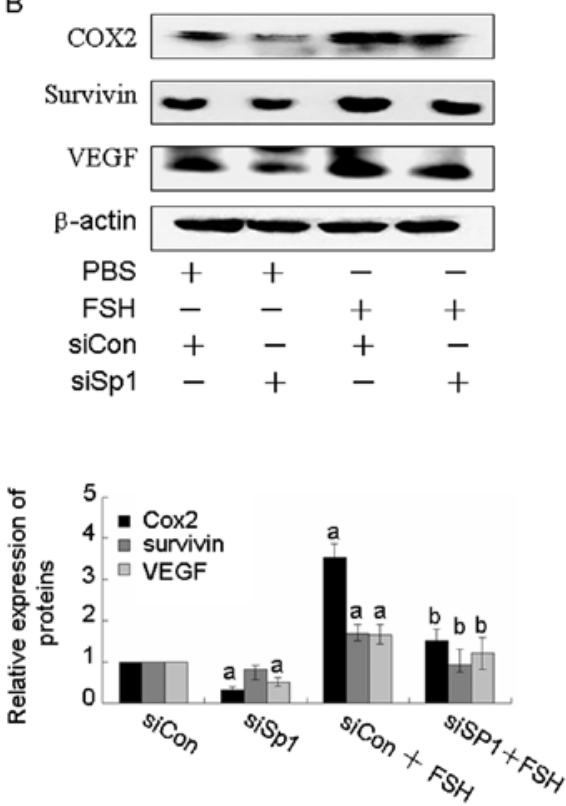
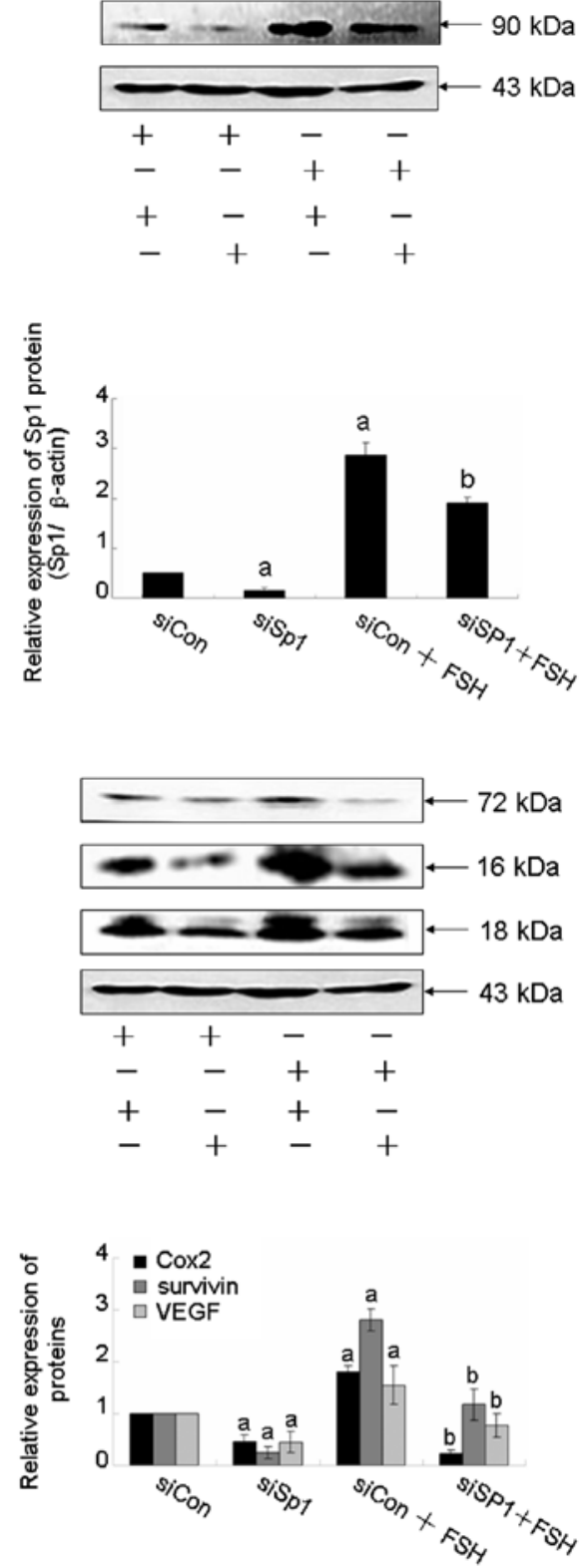

Figure 5. Involvement of Sp1 in FSH-induced VEGF, Cox2 and survivin expression. Hey (left column) and HO8910 (right column) cells were transfected with siSpl and siCon, respectively, after transfection for $12 \mathrm{~h}$, the cells were treated with PBS or $50 \mathrm{mIU} / \mathrm{ml} \mathrm{FSH}$ for another $48 \mathrm{~h}$, western blot analysis was performed to measure (A) Sp1 and (B) VEGF, Cox 2 and survivin expression. ${ }^{a} \mathrm{p}<0.05$ compared with siCon group; ${ }^{\mathrm{b}} \mathrm{p}<0.05$ compared with the group treated with siCon plus $50 \mathrm{mIU} / \mathrm{ml} \mathrm{FSH}$.

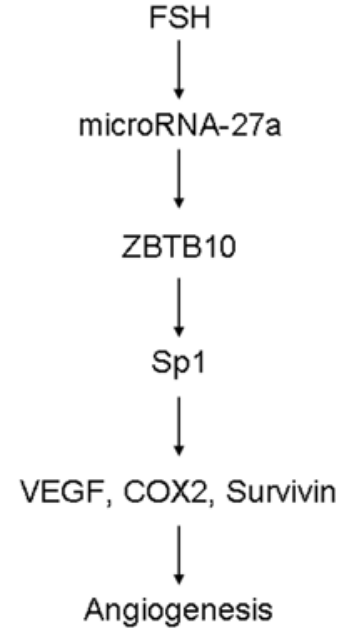

Angiogenesis and HIF1 $\alpha$ via PI3K/AKT signal pathway (4). In this study, we showed the evidence that FSH upregulated not only VEGF, but also Cox 2 and survivin in a dose-dependent manner. Cox 2 plays an important role in regulating VEGF expression, several studies demonstrated that Cox2-mediated VEGF expression might contribute to tumor metastasis via lymphangiogenesis or angiogenesis pathways (19-23). These results imply that overexpression of Cox2, survivin and VEGF facilitates tumor angiogenesis. Although there are positive response between

Figure 6. Proposed signal pathways involved in FSH-induced angiogenesis. FSH may enhance VEGF, Cox 2 and survivin expression through activation of the microRNA-27a: ZBTB10-Sp1 signal pathway, further induces ovarian tumor angiogenesis. 
these three molecule expressions and FSH treatment, the detail mechanism and signal pathway are not clear.

In the current study, it was found that microRNA-27a is required for FSH-induced VEGF, Cox 2 and survivin expression. MicroRNA-27a possesses oncogenic activity, it is involved in cancer development in various tumor types, such as breast and ovarian cancer $(7,24)$. It was found that microRNA-27a overexpressed in several ovarian cancer cell lines, compared with the Moody cell line, a normal ovarian epithelial cell line, which suggests that microRNA-27a is functional in OEC occurrence. In addition, obvious increase in microRNA-27a expression pattern was identified in the present study together with increased dose of FSH. We found that transfection of as-microRNA-27a reduced Cox2, survivin and VEGF expressions, moreover, the induced expressions of the three molecules by FSH were decreased by microRNA-27a, which indicates microRNA-27a contributes to FSH-induced ovarian cancer angiogenesis.

Under rich FSH conditions, reduced ZBTB10 expression was observed. This putative zinc finger protein suppresses specificity protein (Sp) transcription factors and $\mathrm{Sp}$-dependent gene expression $(7,25)$. It was reported that microRNA-27a targets ZBTB10 gene in breast cancer and regulates cell proliferation (7). Our data clearly showed that transfection of as-microRNA-27a induced ZBTB10 expression, the enhanced effect attenuated inhibition of FSH-induced ZBTB10 expression. This is consistent with the results of Mertens-Talcott et al (7). To investigate the role of ZBTB10 on FSH-induced angiogenesis, transfection of ZBTB10 plasmid was performed. Our present result showed that overexpression of ZBTB10 protein inhibited the expressions of VEGF, Cox 2 and survivin, and it abolished FSH-induced expression. These data imply that ZBTB10 plays an opposite role in mediating $\mathrm{FSH}$-induced angiogenesis.

$\mathrm{Sp} 1$ is a critical molecule in microRNA-27a: ZBTB10specificity protein pathway and is a target gene of ZBTB10, which belongs to the $\mathrm{Sp} / \mathrm{krüppel-like} \mathrm{factor} \mathrm{family} \mathrm{of} \mathrm{trans-}$ cription factors (26-28). Previous reports stated that Sp1 was overexpressed in several type of cancer tissues (29-31), moreover, it is a significant predictor of survival in human gastric cancer (30-32). In the current study, overexpression of Sp1 was observed in ovarian cancer cell lines, which indicates that Sp1 may be involved in OEC occurrence. Moreover, the Sp1 protein level was elevated with increased dose of FSH, whereas, transfection of as-microRNA-27a or overexpression of ZBTB10 inhibited Sp1 expression, the inhibition effect abolished FSH-induced Sp1 expression. Present data also clearly showed that RNA interference directed against Sp1 blocked the induction of VEGF, Cox 2 and survivin by FSH, suggesting that Sp1 is a critical molecule in mediating $\mathrm{FSH}$-induced angiogenesis. These results are consistent with previous studies of Sp1 involved in regulation of VEGF expression (33-38).

In conclusion, this study shows that FSH stimulates the micoRNA-27a: ZBTB10-specificity protein pathway to induce VEGF, Cox 2 and survivin expression (Fig. 6), which is the first evidence of direct linkage of FSH to microRNA activation and VEGF, Cox 2 and survivin expression. Our research may help to understand the molecular mechanism of FSH-induced angiogenesis.

\section{Acknowledgements}

The study was supported by grants from the National Natural Science Foundation of China (NSFC no. 81020108027 , no. 30872755 , no. 81172478 ), supported in part by the grant (no. 10JC1413100) from Shanghai Science and Technology Committee.

\section{References}

1. Choi JH, Wong AS, Huang HF and Leung PC: Gonadotropins and ovarian cancer. Endocr Rev 28: 440-461, 2007.

2. Choi KC, Kang SK, Tai CJ, Auersperg N and Leung PC: Follicle-stimulating hormone activates mitogen-activated protein kinase in preneoplastic and neoplastic ovarian surface epithelial cells. J Clin Endocrinol Metab 87: 2245-2253, 2002.

3. Huang Y, Jin H, Liu Y, et al: FSH inhibits ovarian cancer cell apoptosis by up-regulating survivin and down-regulating PDCD6 and DR5. Endocr Relat Cancer 18: 13-26, 2010.

4. Huang Y, Hua K, Zhou X, et al: Activation of the PI3K/AKT pathway mediates FSH-stimulated VEGF expression in ovarian serous cystadenocarcinoma. Cell Res 18: 780-791, 2008.

5. Ryan BM, Konecny GE, Kahlert S, et al: Survivin expression in breast cancer predicts clinical outcome and is associated with HER2, VEGF, urokinase plasminogen activator and PAI-1. Ann Oncol 17: 597-604, 2006.

6. Cai X, Ma S, Gu M, Zu C, Qu W and Zheng X: Survivin regulates the expression of VEGF-C in lymphatic metastasis of breast cancer. Diagn Pathol 7: 52, 2012.

7. Mertens-Talcott SU, Chintharlapalli S, Li X and Safe S: The oncogenic microRNA-27a targets genes that regulate specificity protein transcription factors and the G2-M checkpoint in MDA-MB-231 breast cancer cells. Cancer Res 67: 11001-11011, 2007.

8. Bartel DP: MicroRNAs: genomics, biogenesis, mechanism, and function. Cell 116: 281-297, 2004.

9. Ambros V: The functions of animal microRNAs. Nature 431: 350-355, 2004.

10. Gagan J, Dey BK, Layer R, Yan Z and Dutta A: MicroRNA-378 targets the myogenic repressor MyoR during myoblast differentiation. J Biol Chem 286: 19431-19438, 2011.

11. Kloosterman WP and Plasterk RH: The diverse functions of microRNAs in animal development and disease. Dev Cell 11: 441-450, 2006.

12. Naguibneva I, Ameyar-Zazoua M, Polesskaya A, et al: The microRNA miR-181 targets the homeobox protein Hox-A11 during mammalian myoblast differentiation. Nat Cell Biol 8: 278-284, 2006

13. Sugatani T and Hruska KA: MicroRNA-223 is a key factor in osteoclast differentiation. J Cell Biochem 101: 996-999, 2007.

14. Nishi H, Ono K, Horie T, et al: MicroRNA-27a regulates beta cardiac myosin heavy chain gene expression by targeting thyroid hormone receptor betal in neonatal rat ventricular myocytes. Mol Cell Biol 31: 744-755, 2011.

15. Li X, Mertens-Talcott SU, Zhang S, Kim K, Ball J and Safe S: MicroRNA-27a indirectly regulates estrogen receptor \{alpha\} expression and hormone responsiveness in MCF-7 breast cancer cells. Endocrinology 151: 2462-2473, 2010.

16. Wang J, Luo F, Lu JJ, Chen PK, Liu P and Zheng W: VEGF expression and enhanced production by gonadotropins in ovarian epithelial tumors. Int J Cancer 97: 163-167, 2002.

17. Liao H, Zhou Q, Gu Y, Duan T and Feng Y: Luteinizing hormone facilitates angiogenesis in ovarian epithelial tumor cells and metformin inhibits the effect through the mTOR signaling pathway. Oncol Rep 27: 1873-1878, 2012.

18. Wong C, Wellman TL and Lounsbury KM: VEGF and HIF-1alpha expression are increased in advanced stages of epithelial ovarian cancer. Gynecol Oncol 91: 513-517, 2003.

19. Gallo O, Franchi A, Magnelli L, et al: Cyclooxygenase-2 pathway correlates with VEGF expression in head and neck cancer. Implications for tumor angiogenesis and metastasis. Neoplasia 3: 53-61, 2001

20. Kim MH, Seo SS, Song YS, et al: Expression of cyclooxygenase-1 and -2 associated with expression of VEGF in primary cervical cancer and at metastatic lymph nodes. Gynecol Oncol 90: 83-90, 2003. 
21. Marrogi AJ, Travis WD, Welsh JA, et al: Nitric oxide synthase, cyclooxygenase 2 , and vascular endothelial growth factor in the angiogenesis of non-small cell lung carcinoma. Clin Cancer Res 6: 4739-4744, 2000.

22. Shtivelband MI, Juneja HS, Lee $S$ and Wu KK: Aspirin and salicylate inhibit colon cancer medium- and VEGF-induced endothelial tube formation: correlation with suppression of cyclooxygenase-2 expression. J Thromb Haemost 1: 2225-2233, 2003.

23. Zhang J, Ji J, Yuan F, et al: Cyclooxygenase-2 expression is associated with VEGF-C and lymph node metastases in gastric cancer patients. Biomed Pharmacother 59 (Suppl 2): S285S288, 2005.

24. Li ZM, Hu S, Xiao L, et al: Expression of microRNA 27a and its correlation with drug resistance in human ovarian cancer A2780/Taxol cells. Zhonghua Fu Chan Ke Za Zhi 45: 372-375, 2010 (In Chinese).

25. Chintharlapalli S, Papineni S, Abdelrahim M, et al: Oncogenic microRNA-27a is a target for anticancer agent methyl 2-cyano3,11-dioxo-18beta-olean-1,12-dien-30-oate in colon cancer cells. Int J Cancer 125: 1965-1974, 2009.

26. Philipsen S and Suske G: A tale of three fingers: the family of mammalian Sp/XKLF transcription factors. Nucleic Acids Res 27: 2991-3000, 1999.

27. Bouwman P and Philipsen S: Regulation of the activity of Sp1-related transcription factors. Mol Cell Endocrinol 195: 27-38, 2002.

28. Safe $\mathrm{S}$ and Abdelrahim M: Sp transcription factor family and its role in cancer. Eur J Cancer 41: 2438-2448, 2005.

29. Lou Z, O'Reilly S, Liang H, Maher VM, Sleight SD and McCormick JJ: Down-regulation of overexpressed sp1 protein in human fibrosarcoma cell lines inhibits tumor formation. Cancer Res 65: 1007-1017, 2005.

30. Wang L, Wei D, Huang S, et al: Transcription factor Sp1 expression is a significant predictor of survival in human gastric cancer. Clin Cancer Res 9: 6371-6380, 2003.
31. Zhang J, Zhu ZG, Ji J, et al: Transcription factor Sp1 expression in gastric cancer and its relationship to long-term prognosis. World J Gastroenterol 11: 2213-2217, 2005.

32. Yao JC, Wang L, Wei D, et al: Association between expression of transcription factor Sp1 and increased vascular endothelial growth factor expression, advanced stage, and poor survival in patients with resected gastric cancer. Clin Cancer Res 10: 4109-4117, 2004

33. Akiyama H, Tanaka T, Maeno T, et al: Induction of VEGF gene expression by retinoic acid through Spl-binding sites in retinoblastoma Y79 cells. Invest Ophthalmol Vis Sci 43: 1367-1374, 2002.

34. Bermudez Y, Yang H, Saunders BO, Cheng JQ, Nicosia SV and Kruk PA: VEGF- and LPA-induced telomerase in human ovarian cancer cells is Sp1-dependent. Gynecol Oncol 106: 526-537, 2007.

35. Cho SG, Yi Z, Pang X, et al: Kisspeptin-10, a KISS1-derived decapeptide, inhibits tumor angiogenesis by suppressing Sp1-mediated VEGF expression and FAK/Rho GTPase activation. Cancer Res 69: 7062-7070, 2009.

36. Li ZY, Zhu F, Hu JL, et al: Sp1 inhibition-mediated upregulation of VEGF $165 \mathrm{~b}$ induced by rh-endostatin enhances antiangiogenic and anticancer effect of rh-endostatin in A549. Tumour Biol 32: 677-687, 2011.

37. Loeffler S, Fayard B, Weis J and Weissenberger J: Interleukin-6 induces transcriptional activation of vascular endothelial growth factor (VEGF) in astrocytes in vivo and regulates VEGF promoter activity in glioblastoma cells via direct interaction between STAT3 and Sp1. Int J Cancer 115: 202-213, 2005.

38. Novak EM, Metzger M, Chammas R, et al: Downregulation of TNF-alpha and VEGF expression by Sp1 decoy oligodeoxynucleotides in mouse melanoma tumor. Gene Ther 10: 1992-1997, 2003. 